\title{
Utilización de la Teoría de la Información para evaluar el comportamiento de la estabilidad estática en amputaciones transtibiales
}

\author{
Lely A. Luengas-C. ${ }^{1}$, Daissy C. Toloza $\mathrm{C}^{2}$, Luis F. Wanumen ${ }^{1}$ \\ laluengasc@udistrital.edu.co; daissy.toloza@docentes.umb.edu.co; lwanumen@udistrital.edu.co \\ ${ }^{1}$ Universidad Distrital Francisco José De Caldas, Bogotá, Colombia. \\ ${ }^{2}$ Universidad Manuela Beltrán, Bucaramanga, Colombia.
}

DOI: $10.17013 /$ risti.40.15-30

\begin{abstract}
Resumen: Para examinar la influencia de la amputación en la estabilidad se empleó la teoría de la información (TI), se midió el centro de presión (CoP) de nueve individuos amputados transtibiales y nueve no amputados. Se hizo el análisis de difusión de estabilogramas no lineales y se calcularon medidas infométricas. Los estabilogramas indicaron diferencias en el CoP entre los dos grupos, tanto en dirección antero-posterior (AP) como medial-lateral (ML), siendo mayor el parámetro de la excursión del CoP en los amputados, existe asociación entre la amputación y la estabilidad. La TI dejó en evidencia la incidencia del desplazamiento del CoP en las direcciones AP y ML sobre la estabilidad, en los amputados la mayor afectación de la estabilidad se da AP en el lado no amputado, hay empleo de estrategia de cadera y rodilla para mantener estabilidad postural. Este es el primer reporte del análisis del CoP en amputados transtibiales utilizando TI.
\end{abstract}

Palabras-clave: Amputación transtibial; Estabilidad; Postura; Teoría de la Información.

\section{Use of Information Theory to evaluate the behavior of static stability in transtibial amputations}

\begin{abstract}
Information theory (IT) was used to examine the influence of amputation on stability, the pressure center (CoP) of nine transtibial amputees and nine non-amputees was measured. Diffusion analysis of nonlinear stabilograms was performed and infometric measurements were calculated. Stabilograms indicated differences in CoP between the two groups, in the anteroposterior (AP) and mediallateral (ML) directions, the CoP excursion being higher in amputees, there is an association between amputation and stability. IT revealed the incidence of the CoP displacement in the AP and ML directions on stability, in amputees the greatest affectation of stability occurs AP in the non-amputated side, they use hip and knee strategy to maintain stability postural. This is the first report of $\mathrm{CoP}$ analysis in transtibial amputees using IT.
\end{abstract}

Keywords: Transtibial amputation; Stability; Posture; Information Theory. 


\section{Introducción}

La condición indispensable para realizar actividades diarias que involucran el movimiento de todo el cuerpo, tales como caminar o sentarse se denomina equilibrio postural, y se ha definido como la capacidad de mantener el centro de masa (CM) del cuerpo dentro del polígono de sustentación generado por los pies, esta área es la extensión del casco convexo de los bordes laterales de los pies. La postura de bipedestación estática es inherentemente inestable, ya que los ruidos internos del cuerpo provocan movimientos, pero a través del equilibrio postural las personas tienden a controlar sin esfuerzo la postura para evitar caídas. El incremento de edad, las enfermedades neurodegenerativas, los cambios en estructuras anatómicas, entre otros factores, hacen que el equilibrio se afecte. En los amputados de extremidades inferiores el deterioro del equilibrio aumenta el riesgo de caída (Ihlen, Skjæret, \& Vereijken, 2013; Ku, Azuan, Osman, Abu, \& Wan, 2014; Rhea, Kiefer, Haran, Glass, \& Warren, 2014).

Varios factores conducen a la amputación, como enfermedades, malformaciones congénitas y trauma. La Organización Mundial de la Salud estima que en el mundo alrededor de 30 millones de personas se encuentran amputadas, siendo la amputación transtibial (amputación por debajo de la rodilla) el tipo más común (Luengas C. \& Toloza, 2020b). En Colombia, una causa adicional de las citadas en la amputación transtibial es el trauma por mina antipersonal, a 2020 el Observatorio de Minas de la Vicepresidencia de La República de Colombia registró cerca de 12 mil afectados por estos artefactos, alrededor de 9 mil presentaron heridas y amputaciones y la afectación no cesó durante el confinamiento debido al virus COVID-19 (Dirección Contra Minas, 2020).

En la amputación transtibial unilateral se tiene detrimento de las vías nerviosas, reducción de información somatosensorial, carencia total de la articulación fisiológica del tobillo y de los músculos plantares, ausencia parcial de músculos y huesos de la pierna; todo esto conlleva a una reducción en la movilidad articular y en la fuerza muscular, y compromete la estabilidad, haciendo que se presente alta exposición a las caídas (Curtze, Hof, Postema, \& Otten, 2012; Molero-Sánchez, Molina-Rueda, Alguacil-Diego, Cano-de la Cuerda, \& Miangolarra-Page, 2015). La afectación de una amputación de extremidad inferior se refleja en la disminución de actividad social del amputado, así como en la reducción de la capacidad de locomoción, falta de autonomía y estrés. El propósito fundamental de un programa de rehabilitación protésica es hacer que el amputado recupere y mantenga la destreza para desarrollar actividades diarias usando prótesis, esto se logra si el paciente puede mantener el equilibrio, moverse y caminar al interior y exterior de su espacio de vivienda, sin usar ayudas adicionales. Es imprescindible en este proceso afianzar la confianza de deambulación segura que permita la transferencia de peso sobre cada extremidad, el movimiento de segmentos corporales, longitud de paso y zancada similares entre las extremidades, por citar algunas características. Estudios de estabilidad de personas con amputación transtibial han analizado los parámetros básicos de estabilidad realizando mediciones del centro de presión (CoP) a lo largo del tiempo, así como las estrategias para mantener la estabilidad y la forma de accionar del control postural. Sin embargo, la mayoría de las investigaciones han reclutado pacientes con causas vasculares de amputación, dejando de lado las amputaciones por minas antipersonal (Ku et al., 2014). 
La valoración de la condición física de un sujeto y el monitoreo permanente de su estado permiten establecer el tratamiento terapéutico adecuado, además de determinar su alcance para así conocer el impacto de la terapia y estimar la eficiencia en relación con el costo. Por ello, en el área de la salud es de vital importancia conocer el comportamiento de parámetros asociados a la estabilidad, de allí que se ha hecho uso de manera sistémica de medidas de valoración, donde se incluyen diversos métodos para examinarla. Comúnmente, la caracterización de la estabilidad postural se realiza con la cuantificación del desplazamiento del centro de presión (CoP) en un período de tiempo, se registra con elementos que sensan la fuerza que interactúa entre el pie y la superficie de apoyo y entregan una serie temporal de la posición de la CoP en las direcciones anterior-posterior (AP) y medial-lateral (ML). Las series temporales AP y ML muestran patrones de comportamiento postural, de allí que se analizan de forma independiente con diferentes técnicas tanto en el dominio de tiempo como en frecuencia, pero, estas medidas tienden a ser poco sensibles a la variación y pueden ocultar información relevante de control postural. De allí que se han propuesto otros métodos de análisis, entre los que se encuentran el análisis factorial, información espectral, aplicación de wavelet, por citar algunos (Luengas C. \& Toloza, 2020a; Rhea et al., 2014; Schubert, Kirchner, Dietmar, \& Haas, 2012).

El CoP es el parámetro cuantitativo más estudiado para evaluar la estabilidad postural estática y se define como la fuerza de reacción vertical del suelo que refleja las fuerzas y torques del tobillo necesarios para mantener el centro de gravedad sobre la base de apoyo (Loughlin, Redfern, \& Furman, 2003; Ruhe, Fejer, \& Walker, 2010). La señal COP representa la salida neta del control motor que es necesario para corregir el desequilibrio (Cavanaugh, Guskiewicz, \& Stergiou, 2005; Winter, 1995a), lo que indica que grandes desplazamientos del CoP están asociados con la disminución del control corporal, un indicador de inestabilidad postural y pérdida del equilibrio (Baloh, Corona, Jacobson, Enrietto, \& Bell, 1998; Palmieri, Ingersoll, Stone, \& Krause, 2002; Raymakers, Samson, \& Verhaar, 2005). Se puede visualizar de dos maneras diferentes: a través de un estatocinesiograma o de un estabilograma (Shumway-Cook \& Woollacott, 2007); el estatocinesiograma es el mapa del COP en la dirección antero-posterior (AP) frente al COP en la dirección medio-lateral (ML), es decir, corresponde al área en donde la persona mantiene proyectado su centro de gravedad, mientras que el estabilograma es la serie de tiempo del COP en cada una de las dos direcciones: AP y ML (Pollock, Durward, Rowe, \& Paul, 2000; Winter, 1995b).

En cuanto a su análisis, como lo reportó Toloza et al. (Toloza \& Zequera, 2017) los datos obtenidos del registro del CoP han sido utilizados para calcular un sin número de parámetros o mediciones con el propósito de describir la inestabilidad postural y en los últimos 21 años se han utilizado numerosos parámetros enfocados al análisis lineal en el dominio del tiempo y de la frecuencia (por ejemplo, promedio, valor RMS, amplitud, velocidad, entre otros), pero recientemente se utilizan nuevas técnicas encaminadas al análisis de la señal, las cuales permiten obtener otro tipo de información (Myklebust, Prieto, \& Myklebust, 1995; Palmieri et al., 2002; Raymakers et al., 2005; Shumway-Cook \& Woollacott, 2007; Toloza \& Zequera, 2017), uno de estos métodos es la minería de datos, ya que mostrado alto desempeño en la determinación de enfermedades (Oviedo Carrascal, Sanguino Cotte, Restrepo Arango, \& Patiño Vélez, 2019) y tiene como base las 
Tecnologías de la Información y Comunicaciones (TICs) (Rivas \& Bayona-Oré, 2019). Hasta el momento en la literatura no se han encontrado reportes del análisis del CoP utilizando teoría de la información.

Claude E. Shannon estableció la Teoría Matemática de la Comunicación (A Mathematical Theory of Communication) para relacionar ecuaciones matemáticas con la transmisión de información, detallando la medición de cantidad de información y la capacidad de transmisión del sistema. La teoría de la información (TI) toma como base esta teoría y permite conocer la existencia de relación entre una o varias entradas y la salida en un sistema, utiliza las teorías de la probabilidad para poner los datos en contexto de forma ordenada. Todo sistema de comunicación presenta tres elementos: información, ruido y equivocación; la información concentra las características válidas del resumen que se aplican a los datos. Ruido implica que, en mayor o menor medida, alguna parte de la comunicación expresa conclusiones que pueden parecer sobre los datos, pero que en realidad no son válidos. La equivocación involucra el duplicado de información, parte de la información válida aparece en la comunicación en más de una ocasión, es redundante. Por lo anterior, la información se describe como la comunicación de un resumen con similitudes, diferencias y relaciones descubiertas en los datos, descritas dentro de un contexto particular que incluye características válidas, características erróneas y repetición. El conocimiento está íntimamente relacionado con la información, no se puede informar sin la presencia de un conocimiento, pero se necesita un mínimo de conocimiento para reconocer la información, y se necesita un mínimo de información para obtener un mínimo de conocimiento. Una marcada diferencia entre conocimiento e información es que la información es estática en su transmisión, por otro lado, el conocimiento es dinámico y cambiante, depende del contexto donde se sitúa. La información se contiene en cualquier sistema, independiente de la clase a la cual se suscriba, de allí que se puede usar en la evaluación de datos del ámbito clínico (Shannon, 1948).

La finalidad de esta investigación fue determinar si el uso de TI permite evaluar el comportamiento de la estabilidad de amputados transtibiales unilaterales que sufrieron amputación por trauma de minas antipersonal, a partir de allí establecer la pierna y la dirección de alta injerencia en la estabilidad. Se propuso TI ya que permite descubrir relaciones entre entradas y salidas, sin tener en cuenta el tipo de relación. El poder realizar una evaluación concisa, integral, válida y confiable de la estabilidad en amputados concede herramientas en la toma de decisiones en un contexto clínico.

\section{Método}

\subsection{Experimento}

Se examinó un conjunto de 18 personas, nueve (9) amputados transtibiales y nueve (9) no amputados, clasificados según existencia de amputación en dos grupos: amputado y control. El grupo amputado estuvo compuesto por hombres, presentó un valor medio de edad de $32.35 \pm 3.2$ años, talla $176 \pm 2.7 \mathrm{~cm}$, peso $78.25 \pm 6.5 \mathrm{~kg}$. El grupo control exhibió $44.32 \pm 12.7$ años, $167.44 \pm 8.45 \mathrm{~cm}$ y $69.44 \pm 14.99 \mathrm{~kg}$. Todos era adultos sanos, sin complicaciones físicas ni cognitivas que les impidieran ponerse es posición 
de bipedestación estática. Los amputados usaban prótesis transtibial de suspensión por liner y pin, con pie de alta actividad fabricado en carbono y presentaban independencia en la movilidad. El estudio fue avalado por el Comité de Bioética de la Universidad Distrital Francisco José de Caldas.

El registro de centro de presión (CoP) se llevó a cabo usando plantillas instrumentadas de zapatos, que contienen 198 sensores capacitivos conectados a través de un cable serial a un transmisor inalámbrico para que los datos se trasmitan a un computador y se pueda realizar la adquisición de datos, la señal de cada sensor se muestrea a 50 $\mathrm{Hz}$, el equipo completo es marca Pedar ${ }^{\circledR}$ (Novel.de, 2019). La verificación de correcto funcionamiento del sistema de registro se realizó periódicamente con el dispositivo de calibración 'Trublu' (Novel.de, 2019). El tamaño de plantilla usado fue talla XW que equivale a notación europea 41/42 ya que los participantes se encontraban en esta talla de zapatos. El control de posición de los pies se realizó fijando las plantillas a una superficie plana, con separación de $150 \mathrm{~mm}$ entre puntos medios de talón (Luengas C. \& Toloza, 2020b), esto facilita la postura anatómica de posición bipedestada, donde los brazos están extendidos y relajados al costado y los pies se encuentran uno al lado del otro (Figura 1).

Para el registro de la medición se utilizó el protocolo del Test de Romberg, el cual tiene como objetivo evaluar las habilidades que tiene la persona para mantener la estabilidad postural. Para esta investigación se solicitó a los sujetos participantes adoptar la postura anatómica descrita, en silencio y con los ojos abiertos durante $10 \mathrm{~s}$. Con el fin de minimizar la afectación del rendimiento postural relacionado con la distancia entre los ojos y el campo visual, se indicó que durante el experimento observaran un punto en una pared blanca frente a ellos ubicada a una distancia de $2 \mathrm{~m}$ y estuvieran concentrados en el proceso de control postural para inducir un enfoque interno de atención. Cada prueba se efectuó en tres ensayos, con un tiempo de descanso entre ellos de 5 minutos.

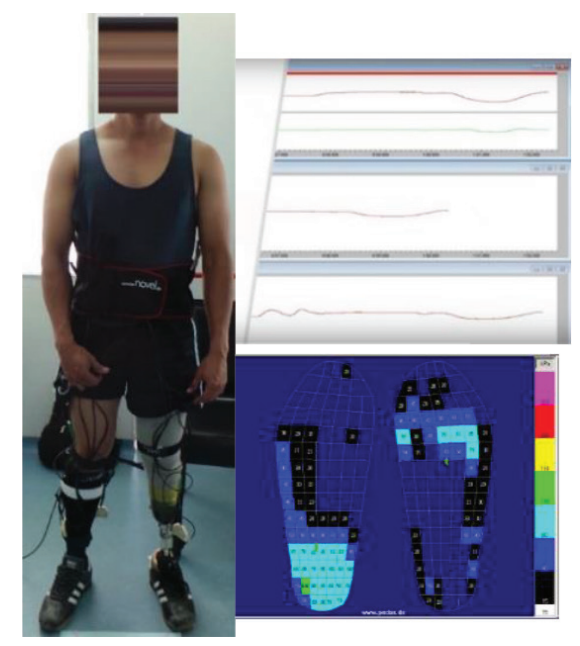

Figura 1 - Medición del centro de presión en un amputado. 


\subsection{Teoría de la Información}

La Teoría de la Información (TI) nace como necesidad de establecer la capacidad de transmisión en los sistemas de comunicaciones, por ello en 1948 Claude Shannon propuso el modelo matemático del proceso de comunicación conocido como la Teoría Matemática de la Comunicación aplicado a un sistema compuesto por señales a transmitir, señales recibidas y canal de comunicación (Figura 2) (Ferreyra, 2007).

El mensaje para transmitir son señales compuestas por distintas variables, el transmisor hace referencia a la información seleccionada, relevante e imprescindible que contiene las características del mensaje y será enviada a través del canal de transmisión. La transferencia de información se afecta por dos fenómenos el ruido y la equivocación; el ruido $\mathrm{H}(\mathrm{Y} \mid \mathrm{X})$ es la incertidumbre de la señal recibida cuando se conoce el mensaje enviado y la equivocación $\mathrm{H}(\mathrm{X} \mid \mathrm{Y}$ ) es la incertidumbre del mensaje enviado cuando se conoce la señal recibida; se observa que tanto el ruido como la equivocación son entropías condicionales que involucran la variable de salida y las variables de entrada. El receptor se encarga de recibir los datos y hacerlos llegar al destinatario. Entonces se puede definir a los datos como una colección de observaciones no analizadas de eventos; la información es el resumen y la comunicación de los principales componentes y relaciones contenidos en los datos y presentados dentro de un contexto específico; el conocimiento es la colección interrelacionada de procedimientos para actuar hacia resultados particulares con referencias asociadas (Ferreyra, 2007; Luengas C., 2016).

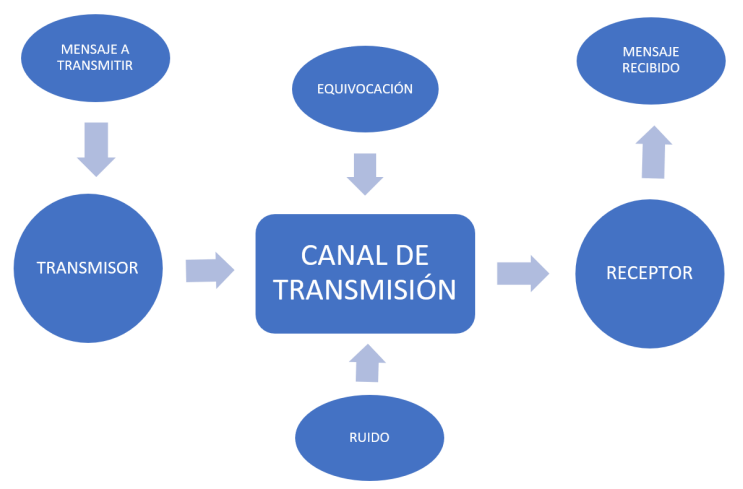

Figura 2 - Sistema de comunicación.

Shannon definió el término información como el conjunto de datos seleccionados, supervisados, ordenados, con características significativas que permite la construcción de un mensaje teniendo como base un suceso; adicional, entregó mecanismos para la medición de la información y así conocer la máxima cantidad de información sin ruido que puede ser transmitida. El término información se relaciona tanto con la cantidad de mensajes dispuestos para transmitir como con la libertad de elección de estos. La cantidad de información de un mensaje hace referencia a la probabilidad de recepción de este, por ello involucra la incertidumbre y usa la entropía $\mathrm{H}(\mathrm{X})$ como medida, 
Ecuación 1; la medida de la $\mathrm{H}(\mathrm{X})$ se da en bits, contiene toda la información incluyendo la señal más el ruido, la probabilidad de selección de un dato es $p_{i} p_{i}$. La medición de información permite descubrir las relaciones existentes entre entradas y salidas, lo que admite la viabilidad de construcción de modelos predictivos, así como la detección de las variables de entrada que efectivamente inciden en la salida (Ferreyra, 2007; Luengas C., 2016).

$$
H(X)=-\Sigma_{i}^{n}=1 p_{i} \log _{2}\left(p_{i}\right)
$$

La relación existente entre una entrada X y la salida Y se puede conocer al calcular la información mutua $\mathrm{I}(\mathrm{X}: \mathrm{Y})$, de esta forma se establece la cantidad de información neta transmitida, que depende de la información transmitida y se ve afectada por el ruido y la equivocación, Ecuación 2. Existe relación entre entradas y salidas siempre y cuando $\mathrm{I}(\mathrm{X}: \mathrm{Y})$ sea mayor a cero (Luengas C., 2016; Pyle, 2003).

$$
I(X: Y)=H(X)-H(X \mid Y)=H(Y)-H(Y \mid X)
$$

El empleo de TI para analizar datos requiere que estos sean categóricos, cuando son de tipo numérico se aplican algoritmos de agrupación con el fin de crear conjuntos limitados (en inglés bins), lo cual conlleva a la reducción de ruido y al incremento de la señal. Un algoritmo de tipo supervisado es el llamado Menor Pérdida de Información (LIL), toma como base la TI para situar los datos en determinado grupo según la cantidad de pérdida de información de la salida, de esta forma maximiza la cantidad de información sobre la salida acorde con el número de categorías. La determinación de las categorías se realiza con diversos métodos, entre ellos la relación señal/ruido que utiliza TI para reducir el ruido y maximizar la cantidad de información; se debe asegurar que las agrupaciones y variables elegidas sean representativas del sistema (Gabrys, Leiviskä, \& Strackeljan, 2005; Kotsiantis \& Kanellopoulos, 2006; Pyle, 1999).

\subsection{Análisis de datos}

Una vez obtenido el registro de los datos del CoP, se calculó el desplazamiento de este en cada pie (izquierdo y derecho) en los ejes antero-posterior (AP) y medial-lateral (ML), de esta forma se tiene para cada sujeto cuatro variables: antero-posterior lado izquierdo (YL), medial-lateral lado izquierdo (XL), antero-posterior lado derecho (YR) y mediallateral lado derecho (XR). Para reducir el ruido de las mediciones se aplicó un filtro Butterworth pasa bajo de cuarto orden con una frecuencia de corte de $10 \mathrm{~Hz}$ y se realizó reducción de tendencia (en inglés detrended) por la media de la serie de tiempo. La frecuencia de corte se elige teniendo en cuenta que cerca del 95\% de la información se encuentra en un rango bajo esta frecuencia (Hay \& Wachowiak, 2017; Schubert et al., 2012). Se procedió a la obtención de las gráficas de estabilograma y el estatocinesiogra para observar el comportamiento temporal de la estabilidad tanto en amputados como en control (Hendershot \& Nussbaum, 2013; Schubert et al., 2012). Para este análisis se calculó la excursión, la velocidad promedio, el rango y la amplitud promedio, se empleó la estadística U de Mann-Whitney ( $\mathrm{p}<0.05)$ para evaluar los dos grupos de 
trabajo. Igualmente, se evaluó la distribución normal de estos parámetros con la prueba estadística de Kolmogorov-Smirnoff $(\mathrm{p}<0.05)$. Estos análisis fueron realizados con el software SPSS20.0.

El patrón de estabilidad en cada grupo se evaluó usando teoría de la información (TI), una restricción de TI es la resolución finita de datos a examinar, para ello se aplicó el método de agrupación Menor Pérdida de Información (LIL, por sus siglas en inglés) en las cuatro variables, convirtiendo los datos numéricos de las mediciones en datos categóricos. Con los datos categóricos se observó la influencia de cada variable sobre la estabilidad, obteniendo las cantidades infométricas del sistema y determinando los parámetros que favorecen la maximización de las características de este sistema y reducen la información errónea (ruido), para ello se empleó el algoritmo de Mayor Información. En este se seleccionan las variables que transmiten la mayor cantidad de información y las más representativas de la salida, es decir que proporcionen la mayor ganancia (totalidad de información transmitida), el orden de selección es descendiente y depende de la cantidad de información que cada una contiene, si alguna variable incluida inicialmente en el análisis no aporta información no será tenida en cuenta (Luengas C., 2016). La inspección de datos con TI se realizó con el software Powerhouse Analytics 2.2.

Una vez se tienen los datos categóricos y las variables seleccionadas se realiza el cálculo de la cantidad de información media de los mensajes, es decir $\mathrm{H}(\mathrm{X})$, también se obtiene la entropía condicional de la entrada con respecto a la salida y de la salida con respecto a la entrada para así determinar el valor de equivocación que se puede presentar en el sistema y el ruido generado por cada una de las variables y del conjunto de las variables. Estos valores permiten conocer la cantidad de información transmitida o la información mutua I(X:Y).

Adicional, se indagó en los patrones y relaciones existentes en los datos, usando un modelo explicativo basado en Clúster, agrupando los datos en registros con características similares y que se distinguen de otras agrupaciones. El análisis usando clústeres permite clasificar todo un conjunto de datos en subconjuntos homogéneos en información usando técnicas multivariantes siempre teniendo como base una estructura entrópica interna, ya que los componentes entrópicos no son sensibles al modo de transmisión de la información, consideran componentes no lineales y con presencia de ruido. Los registros o agrupaciones se visualizan en un mapa de densidad, cada registro se proyecta en un espacio de información donde la distancia entre registros es proporcional a la similitud del contenido de información, esto hace que se presente variación en la densidad de cada grupo, los puntos más densos de cada espacio conforman el centro del clúster y los menos densos son los bordes. De forma gráfica la densidad se representa por colores: existen puntos negros que indican el lugar de un registro; el rojo se usa al existir alta densidad con gran cantidad de información similar entre sí; el azul indica baja densidad con pocos registros; los valores intermedios de densidad usan el color verde, amarillo o naranja. Los clústeres se descubren al encontrar la mayor separación de las áreas de alta densidad.

\section{Resultados y análisis}

La observación del comportamiento de estabilidad en los grupos estudiados inició con la obtención de las gráficas propias de estabilidad, como son el estabilograma 
y el estatocinesiograma (Figura 3). Los amputados exhiben mayor variación en los dos ejes y en ambas piernas en comparación con el grupo control. Lo anterior se confirma con los resultados de los parámetros calculados de la excursión, la velocidad promedio, el rango y la amplitud promedio en ambos grupos. En la Tabla 1 se observa que todos los parámetros difieren en ambos grupos, siendo los mayores valores en el grupo de amputados en ambas direcciones (ML y AP). Al desglosar por los ejes se observó que la dirección AP presentó el mayor desplazamiento con respecto al ML en el grupo de los amputados. Todos los valores presentaron diferencias estadísticamente significativas.

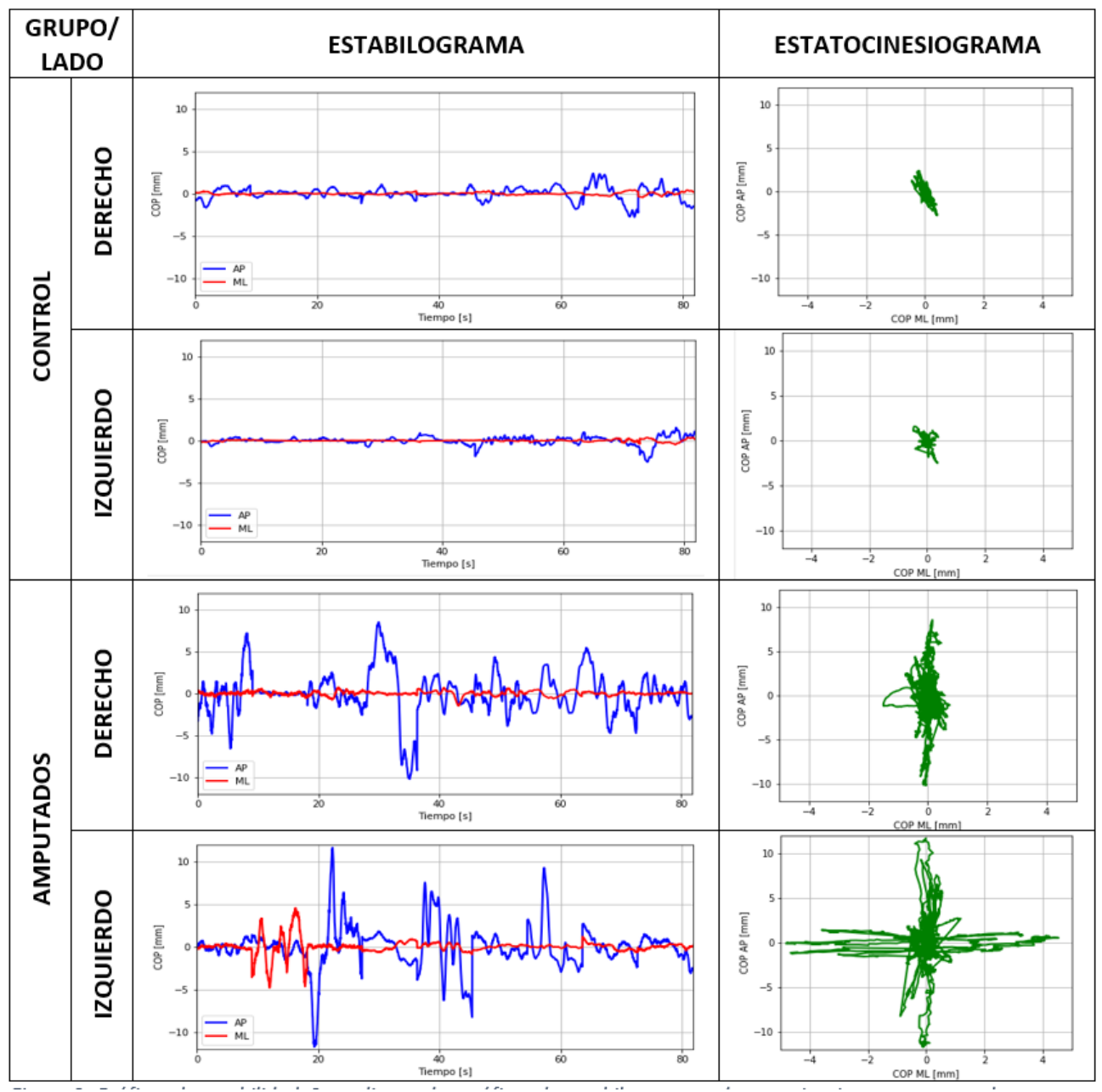

Figura 3 - Gráficas de estabilidad. Se realizaron las gráficas de estabilograma y el estatocinesiograma para cada grupo. 


\begin{tabular}{|c|c|c|c|c|c|c|c|c|}
\hline \multirow[t]{3}{*}{ Parámetros } & \multicolumn{4}{|c|}{ Amputados } & \multicolumn{4}{|c|}{ Control } \\
\hline & \multicolumn{2}{|c|}{ PI } & \multicolumn{2}{|c|}{ PD } & \multicolumn{2}{|c|}{ PI } & \multicolumn{2}{|c|}{ PD } \\
\hline & ML & AP & ML & $\mathbf{A P}$ & ML & $\mathbf{A P}$ & ML & AP \\
\hline Excursión (mm) & 68,89 & 1134,3 & 214,94 & 436,28 & 30,62 & 152,11 & 43,35 & 246,55 \\
\hline Velocidad (mm/s) & 0,91 & 5,38 & 1,39 & 2,20 & 0,18 & 0,77 & 0,19 & 0,98 \\
\hline Rango (mm) & 0,83 & 12,01 & 2,21 & 4,24 & 0,32 & 1,71 & 0,42 & 2,51 \\
\hline $\begin{array}{l}\text { Amplitud promedio } \\
(\mathrm{mm})\end{array}$ & 0,15 & 2,50 & 0,47 & 0,96 & 0,07 & 0,34 & 0,10 & 0,54 \\
\hline
\end{tabular}

Tabla 1 - Valores promedio del CoP

En la Figura 4 se puede observar la comparación de los parámetros calculados en los dos grupos, se ratifica que los amputados presentan un desplazamiento con mayor predominancia hacia el eje AP específicamente en la pierna izquierda, en todos los parámetros calculados. Así mismo, se observa que las mayores diferencias entre los dos grupos de análisis se presentaron en las medidas de la excursión y el rango, lo que indican que la población de amputados transtibiales en posición bípeda son más inestables, con una oscilación mayor en la dirección antero-posterior, motivo por el cual, los hace más propensos a sufrir una caída. Igualmente, lo obtenido en el estabilograma y estatocinesiograma (Figura 3) corrobora los parámetros calculados (Figura 4). En los amputados el CoP se ubica medial y anterior en el lado amputado en comparación con el lado no amputado, esto puede suceder como consecuencia del tipo de pie protésico(Luengas C. \& Toloza, 2020a).

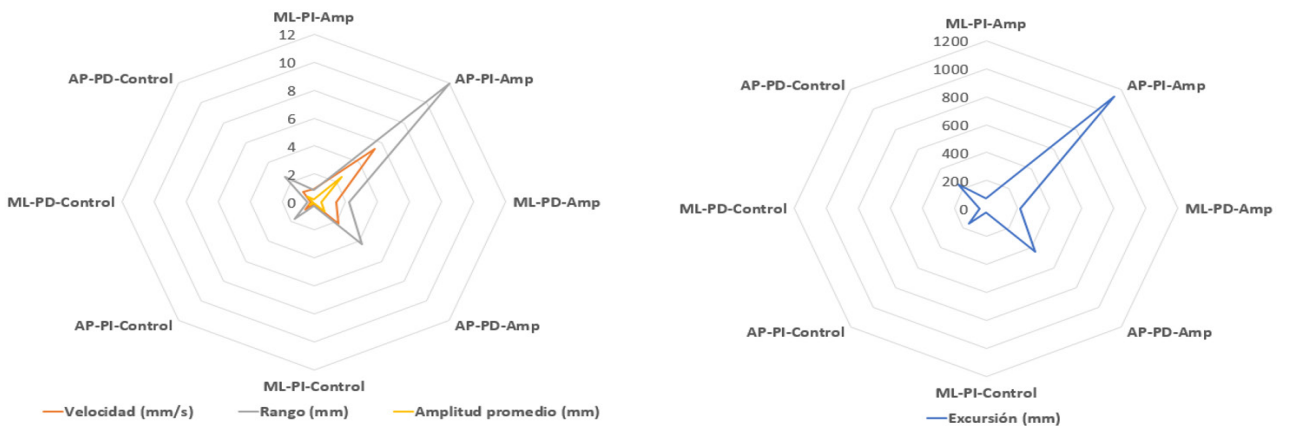

Figura 4 - Valores promedio de los parámetros calculados. ML: Mediolateral - AP: Anteroposterior - PI: Pierna izquierda - PD: Pierna derecha - Amp: Amputados

Al realizar el examen de la información del sistema se observó que todas variables presentaron alto contenido de información, así como gran cantidad de datos diferentes en cada una de ellas. Luego de aplicar LIL se consiguió que cada variable contuviera 10 
agrupaciones o bins, lo cual redujo considerablemente el número de datos distintos, afectando los parámetros estadísticos (Tabla 2) pero teniendo valores representativos de la población con un índice general de confianza superior al 85\% (Tabla 3). La cuantía de valores distintos en cada variable se relaciona con la cuantía de información transportada de la entrada a la salida, al obtener los bins la cantidad de información disminuye, pero se contienen las características del sistema.

\begin{tabular}{llccccc}
\hline \multicolumn{2}{c}{ PARÁMETRO } & Máximo & Media & Mediana & Mínimo & Cantidad datos diferentes \\
\hline \multirow{2}{*}{ XL } & INICIAL & 0,747 & 0,004 & 0,004 & $-1,489$ & 948 \\
\cline { 2 - 7 } YL & LIL & 0,747 & 0 & 0,003 & $-1,493$ & 10 \\
\cline { 2 - 7 } & LIL & 8,519 & 0,015 & 0,023 & $-10,221$ & 2706 \\
\hline \multirow{2}{*}{ XR } & INICIAL & 4,519 & 0 & 0,020 & $-10,221$ & 10 \\
\cline { 2 - 7 } & LIL & 4,503 & 0,001 & $-0,008$ & $-4,784$ & 1255 \\
\hline
\end{tabular}

Tabla 2 - Valores estadísticos de las variables de entrada. Inicial son los estadísticos de los datos numéricos originales de medición, LIL son los estadísticos de los datos agrupados

Debido al tipo de sistema que se tiene en el presente estudio, un sistema realimentado, se determinó el aporte de cada variable a la estabilidad (Tabla 3) así como la ganancia otorgada por el conjunto de variables (Tabla 3). Todas las variables involucradas al inicio de la investigación fueron seleccionadas, es decir todas aportan información al sistema de estabilidad.

\begin{tabular}{llllll}
\hline VARIABLE & XL & YL & XR & YR & TOTAL \\
\hline APORTE (\%) & 9,01 & 32,88 & 18,07 & 16,63 & $\mathbf{7 6 , 5 9}$ \\
\hline CONFIANZA (\%) & 85 & 96 & 95 & 94 & $\mathbf{8 5 , 3 7}$ \\
\hline
\end{tabular}

Tabla 3 - Datos infométricos de las variables estudiadas

El mapa infométrico del sistema de estabilidad (Figura 5) muestra que la cantidad de información ingresada al sistema a partir de los datos de las variables es de 7,29 bits y corresponde al $77 \%$ del máximo teórico de información posible que todos los datos podrían contener, aquí se incluye tanto información real como duplicada; la información transmitida es 0,77 bits concerniente al 77\% de la información requerida para representar la salida, de esta forma la incertidumbre acerca de la salida se reduce; el ruido de 0,23 bits transmite $23 \%$ de información improductiva, que no colabora para reducir la incertidumbre acerca de los estados de la variable de salida; la equivocación de 6,52 bits, muestra que el 89\% de la información en los datos de entrada está repetida. 


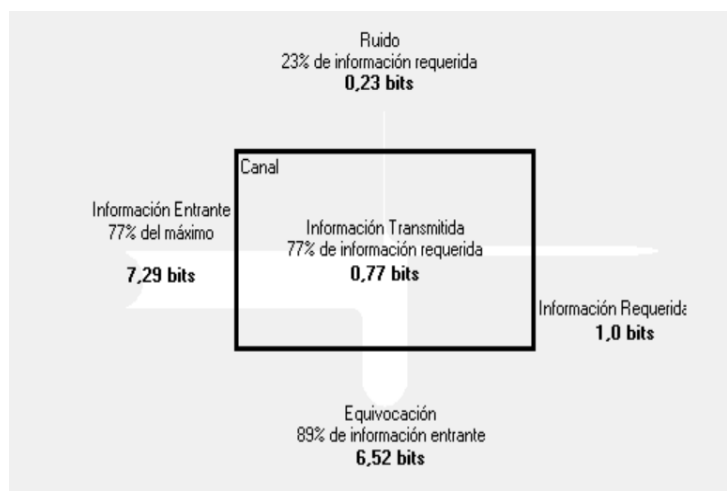

Figura 5 - Mapa infométrico del sistema de estabilidad

\section{Conclusiones}

La exploración detallada de las variables del CoP en el estabilograma y el estatocinesiograma reveló una alta variación del desplazamiento en los amputados, así como valores mayores, tanto en dirección antero-posterior como medial-lateral, siendo la más alta presentada por el lado no amputado en antero-posterior. Esto demuestra la existencia de baja estabilidad en los amputados en relación con los no amputados, así como el uso continuo y superior de estrategias de control postural. Lo anterior comprueba que los sujetos con integración sensorimotora alterada, tales como los amputados transtibiales, presentan perturbación en la estabilidad, ya que se reduce el control voluntario de la ubicación del CoP con respecto a la base, debido a la pérdida de todas las entradas externas causada por la amputación.

El no poseer tobillo anatómico, ni tener la información propioceptiva completa, así como presentar alteración en la morfología del cuerpo entero, hace que el centro de masa se desplace e influya en la ubicación del centro de presión (CoP), además, los parámetros propios de la estabilidad (excursión, velocidad, rango y amplitud promedio) se ven afectados. Lo que significa que hacen uso continuo y con mayor frecuencia de las estrategias de compensación para mantener la estabilidad en la posición de bipedestación. Igualmente, La diferencia en el patrón de balanceo sugiere que los cambios en el desplazamiento anteroposterior del COP podrían ser una fuente predictiva, o incluso un requisito para la detección correcta del movimiento en amputados.

El mapa de información (Figura 4) muestra que las variables de entrada llevan un total de 7,29 bits de información, donde se tienen 6,52 bits en equivocación, es decir información duplicada que no aporta, luego la información útil que da cuenta de las características del sistema es de 0,766 bits. La entropía de la variable de salida indica que se necesita 1 bit para predecirla con total exactitud, pero se reduce debido a la inclusión de ruido y equivocación. La existencia de un valor superior a o\% de información transmitida indica la presencia de una asociación entre las variables de entrada y la salida, sin distinguir el tipo de relación, es decir comprueba la relación existente entre ubicación del CoP en cada pie y la estabilidad. Al examinar individualmente cada variable, YR involucra 
la mayor cantidad de ruido al sistema, mientras que YL aporta el mínimo; pero, al realizar el examen en forma conjunta de las variables es YL la que involucra la más alta información no pertinente al sistema.

La estabilidad en el sistema estudiado está afectada por las cuatro variables seleccionadas YL, XR, YR y XL, es decir el desplazamiento del COP en cada pie y cada dirección se relaciona con la estabilidad, pero en diferente medida; las variables en conjunto aportan 0,7658 bits, lo que equivale al $77 \%$ de la información requerida para establecer la existencia de estabilidad. La variación antero-posterior del lado no amputado es la variable de mayor incidencia en la estabilidad, con un aporte de 32,88\% y nivel de confianza de $96 \%$, la de menor incidencia es la variación medial-lateral del lado no amputado, aporte de 9,01\% y confianza de 85\%, esto concuerda con resultados de otros investigadores (Rougier \& Bergeau, 2009) Nuestros hallazgos revelan la existencia de mecanismos de compensación para mantener la estabilidad usados recurrentemente en el lado no amputado, los cuales pueden ser estrategia de cadera y de rodilla asociados a la variación antero-posterior, inhibiendo el uso de tobillo, el cual está vinculado a la variación medial-lateral (Hlavackova, Franco, Diot, \& Vuillerme, 2011; Luengas C. \& Toloza, 2020b).

El tener un nivel de confianza superior al 85\% en cada variable implica que la técnica empleada para la reducción del número de datos y la conversión de tipo numéricos a categóricos fue eficiente, entregando valores representativos de la población.

Los resultados arrojados al realizar la exploración usando TI coinciden con lo obtenido en otros métodos empleados, tales como análisis frecuencial y transformada wavelet (Luengas C. \& Toloza, 2020a, 2020b), esto muestra que la teoría de la información se puede emplear como una herramienta para el análisis postural.

\section{Referencias}

Baloh, R. W., Corona, S., Jacobson, K. M., Enrietto, J. A., \& Bell, T. (1998). A prospective study of posturography in normal older people. Journal of the American Geriatrics Society, 46(4), 438-443.

Cavanaugh, J. T., Guskiewicz, K. M., \& Stergiou, N. (2005). A nonlinear dynamic approach for evaluating postural control: new directions for the management of sport-related cerebral concussion. Sports Medicine (Auckland, N.Z.), 35(11), 935-950. http://dx.doi.org/10.2165/00007256-200535110-00002

Curtze, C., Hof, A. L., Postema, K., \& Otten, B. (2012). The relative contributions of the prosthetic and sound limb to balance control in unilateral transtibial amputees. Gait and Posture, 36(2), 276-281. https://doi.org/10.1016/j.gaitpost.2012.03.010

Dirección Contra Minas. (2020). Víctimas de minas antipersonal y municiones sin explosionar. http://www.accioncontraminas.gov.co/Estadisticas/Paginas/ Estadisticas-de-Victimas.aspx

Ferreyra, M. (2007). Powerhouse: Data Mining usando Teoría de la información. http://web.austral.edu.ar/images/contenido/facultad-ingenieria/2-Data_ Mining_basado_Teoria_Informacion_Marcelo_Ferreyra.pdf 
Gabrys, B., Leiviskä, K., \& Strackeljan, J. (2005). Do Smart Adaptive Systems Exist?. Polonia: Springer.

Hay, D. C., \& Wachowiak, M. P. (2017). Analysis of free moment and center of pressure frequency components during quiet standing using magnitude squared coherence. Human Movement Science, 54, 101-109. https://doi.org/10.1016/J. HUMOV.2017.04.002

Hendershot, B. D., \& Nussbaum, M. A. (2013). Persons with lower-limb amputation have impaired trunk postural control while maintaining seated balance. Gait \& Posture, 1-5. https://doi.org/10.1016/j.gaitpost.2013.01.008

Hlavackova, P., Franco, C., Diot, B., \& Vuillerme, N. (2011). Contribution of each leg to the control of unperturbed bipedal stance in lower limb amputees: New insights using entropy. PLoS ONE, 6(5), 1-4. https://doi.org/10.1371/journal.pone.0019661

Ihlen, E. A. F., Skjæret, N., \& Vereijken, B. (2013). The influence of center-of-mass movements on the variation in the structure of human postural sway. Journal of Biomechanics, 46(3). https://doi.org/10.1016/j.jbiomech.2012.10.016

Kotsiantis, S., \& Kanellopoulos, D. (2006). Discretization techniques: A recent survey. GESTS International Transactions on Computer Science and Engineering, 32(1), 47-58.

Ku, P. X., Azuan, N., Osman, A., Abu, W., \& Wan, B. (2014). Balance control in lower extremity amputees during quiet standing : A systematic review. Gait \& Posture, $39,672-682$.

Loughlin, P. J., Redfern, M. S., \& Furman, J. M. (2003). Nonstationarities of Postural Sway. IEEE Engineering in Medicine and Biology Magazine, 22(2), 69-75. https://doi.org/10.1109/MEMB.2003.1195699

Luengas C., L. A. (2016). Modelo de alineación estática para prótesis transtibiales. Pontificia Universidad Javeriana.

Luengas C., L. A., \& Toloza, D. C. (2020a). Application of wavelet transform to stability analysis in transtibial amputees. Investigación e Innovación En Ingenierías, 8(1), 214-225. https://doi.org/10.17081/invinno.8.1.3640

Luengas C., L. A., \& Toloza, D. C. (2020b). Frequency and Spectral Power Density Analysis of the Stability of Amputees Subjects. TecnoLógicas, 23(48), 1-16. https://doi.org/10.22430/22565337.1453

Molero-Sánchez, A., Molina-Rueda, F., Alguacil-Diego, I. M., Cano-de la Cuerda, R., \& Miangolarra-Page, J. C. (2015). Comparison of stability limits in men with traumatic transtibial amputation and nonamputee control group. $P M$ and $R, 7(2), 123-129$. https://doi.org/10.1016/j.pmrj.2014.08.953

Myklebust, J. B., Prieto, T., \& Myklebust, B. (1995). Evaluation of nonlinear dynamics in postural steadiness time series. Annals of Biomedical Engineering, 23(6), 711-719. https://doi.org/10.1007/BFo2584470 
Novel.de. (2019). The pedar ${ }^{\circledR}$ system. http://www.novel.de/novelcontent/pedar

Oviedo Carrascal, A. I., Sanguino Cotte, D., Restrepo Arango, N. A., \& Patiño Vélez, A. F. (2019). Descubrimiento de Conocimiento en Historias Clínicas mediante Minería de Texto. RISTI - Revista Ibérica de Sistemas e Tecnologias de Informação, (34), 29-43. https://doi.org/10.17013/risti.34.29-43

Palmieri, R. M., Ingersoll, C. D., Stone, M. B., \& Krause, B. A. (2002). Center-ofPressure Parameters Used in the Assessment of Postural Control. Journal of Sport Rehabilitation, 11(1), 51-66. https://doi.org/10.1123/jsr.11.1.51

Pollock, A. S., Durward, B. R., Rowe, P. J., \& Paul, J. P. (2000). What is balance? Clinical Rehabilitation, 14(4), 402-406. https://doi.org/10.1191/0269215500cr3420a

Pyle,D.(1999).Datapreparationfordatamining.MorganKaufmannPublishers,Inc.San Francisco: Morgan Kaufmann Publishers, Inc. https://doi.org/10.1080/713827180

Pyle, D. (2003). Business Modeling and Data Mining. San Francisco: Morgan Kaufmann Publishers, Inc. https://doi.org/10.1016/B978-155860653-1.50012-9

Raymakers, J. A., Samson, M. M., \& Verhaar, H. J. J. (2005). The assessment of body sway and the choice of the stability parameter(s). Gait and Posture, 21(1), 48-58. https://doi.org/10.1016/j.gaitpost.2003.11.006

Rhea, C. K., Kiefer, A. W., Haran, F. J., Glass, S. M., \& Warren, W. H. (2014). A new measure of the CoP trajectory in postural sway: Dynamics of heading change. Medical Engineering and Physics, 36(11), 1473-1479. https://doi.org/10.1016/j. medengphy.2014.07.021

Rivas, M. H., \& Bayona-Oré, S. (2019). Algoritmos de Minería de Proceso para el Descubrimiento Automático de Procesos. RISTI - Revista Ibérica de Sistemas e Tecnologias de Informação, (31), 33-49. https://doi.org/10.17013/risti.31.33-49

Rougier, P. R., \& Bergeau, J. (2009). Biomechanical Analysis of Postural Control of Persons with Transtibial or Transfemoral Amputation. American Journal of Physical Medicine \& Rehabilitation, 88(11), 896-903. https://doi.org/10.1097/ PHM.obo13e3181b331af

Ruhe, A., Fejer, R., \& Walker, B. (2010). The test-retest reliability of centre of pressure measures in bipedal static task conditions - A systematic review of the literature. Gait and Posture, 32(4), 436-445. https://doi.org/10.1016/j.gaitpost.2010.09.012

Schubert, P., Kirchner, M., Dietmar, S., \& Haas, C. T. (2012). About the structure of posturography: Sampling duration, parametrization, focus of attention (part I). J. Biomedical Science and Engineering, 5, 496-507. https://doi.org/10.4236/ jbise.2012.59062

Shannon,C.E.(1948).Amathematicaltheoryofcommunication.TheBellSystem Technical Journal, 27(July 1928), 379-423. https://doi.org/10.1145/584091.584093

Shumway-Cook, A., \& Woollacott, M. H. (2007). Motor control : translating research into clinical practice. Lippincott Williams \& Wilkins. 
Toloza, D., \& Zequera, M. (2017). Linear and non-linear methods for analysis center pressure and its application in diabetic peripheral neuropathy: A systematic review. In IFMBE Proceedings (Vol. 6o). https://doi.org/10.1007/978-981-10-4086-3_179

Winter, D. A. (1995a). A.B.C. (anatomy, biomechanics and control) of balance during standing and walking. Waterloo Biomechanics.

Winter, D. A. (1995b). Human blance and posture control during standing and walking. Gait \& Posture, 3(4), 193-214. https://doi.org/10.1016/o966-6362(96)82849-9 